\title{
The Journal of
}

COLLEGIATE EMERGENCY MEDICAL SERVICES

\section{Research and Scholarship in Collegiate Emergency Medical Services: Current State and Opportunities for Progress}

Nicholas M.G. Friedman; Brittany J. Dingler; Jose V. Nable; George J. Koenig

Keywords: collegiate emergency medical services; campus-based pre-hospital emergency care; research; scholarship; journal

Citation (AMA Style): Friedman NMG, Dingler BJ, Nable JV, Koenig GJ. Research and Scholarship in Collegiate Emergency Medical Services: Current State and Opportunities for Progress. J Coll Emerg Med Serv. 2018; 1(1): 5-12. https:// doi.org/10.30542/JCEMS.2018.01.01.01

Electronic Link: https://doi.org/10.30542/JCEMS.2018.01.01.01

Published Online: February 23, 2018

Published in Print: February 23, 2018 (Volume 1: Issue 1)

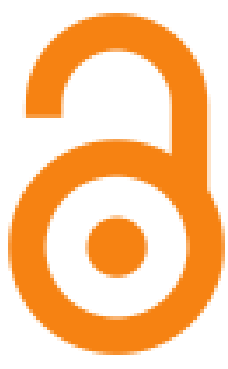

Copyright: (C) 2018 Friedman, Dingler, Nable, \& Koenig. This is an OPEN ACCESS article distributed under the terms of the Creative Commons Attribution 4.0 International (CC BY 4.0) License, which permits unrestricted use, distribution, and reproduction in any medium, provided the original author and source are credited. The full license is available at: https://creativecommons.org/licenses/by/4.0/ 


\title{
Research and Scholarship in Collegiate Emergency Medical Services: Current State and Opportunities for Progress
}

\author{
JCEMS and NCEMSF are spearheading the drive to promote research and scholarship \\ in the field of campus-based pre-hospital emergency care.
}

Nicholas M.G. Friedman, BA, EMT-B; Brittany J. Dingler, BA, PA-S; Jose V. Nable, MD, MS, NRP; George J. Koenig, DO, MS

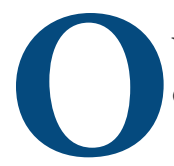
ver the last twenty-five years, the collegiate or campus-based emergency medical services (CBEMS) community has evolved at an impressive rate, and new CBEMS organizations are instituted nearly every year. Although organizations dedicated to campus-based EMS trace back to at least 1948', the establishment of the National Collegiate EMS Foundation (NCEMSF) in 1993 marks a seminal event in the history of collegiate EMS. Prior to the establishment of NCEMSF, CBEMS organizations struggled to efficiently share resources and network with each other. Further inhibiting the advancement of collegiate EMS, a bias towards CBEMS organizations existed within the broader EMS community. Despite the fact that CBEMS organizations staffed licensed EMS providers, there was

Nicholas M.G. Friedman, BA, EMT-B is the Editor-in-Chief of JCEMS and the former director of Skidmore College EMS. Brittany J. Dingler, BA, PA-S is the Executive Editor of JCEMS and a student in the Physician Assistant program at Drexel University. Jose V. Nable, MD, MS, NRP is an Assistant Professor of Emergency Medicine at Georgetown University School of Medicine and the medical director of Georgetown Emergency Response Medical Service. George J. Koenig, DO, MS is an Assistant Professor of Surgery in the Division of Acute Care Surgery: Trauma, Surgical Critical Care and Emergency General Surgery of Sidney Kimmel Medical College at Thomas Jefferson University and the President of the National Collegiate EMS Foundation.
Key Points

Problem: There is a lack of CBEMS-related research and scholarship in the literature.

Opportunity: Situated at academic institutions, CBEMS organizations are well positioned to conduct research and to produce publishable scholarship.

Progress In partnership with NCEMSF, JCEMS developed the first peer-reviewed journal in the field of campus-based pre-hospital emergency care. JCEMS and NCEMSF seek to motivate and support research efforts.

a perception that CBEMS organizations provided a lower quality of care than traditional EMS services. To promote the advancement of collegiate EMS, NCEMSF facilitated communication between organizations and developed a robust network of supportive alumni and professionals. In addition, through education and advocacy, NCEMSF provided CBEMS organizations with the legitimacy needed to gain the approval of the EMS community. Without question, NCEMSF has markedly progressed the field of college health and safety.

Yet, notwithstanding the opportunities facilitated by NCEMSF, a gap exists in the scholarly literature 
and in popular sources on CBEMS. In this editorial, our aim is to motivate research and other scholarly efforts within the CBEMS community. We will articulate the gap in the scholarly literature and identify why CBEMS organizations are well positioned to overcome the barriers associated with EMS research. We will then discuss how NCEMSF and The Journal of Collegiate Emergency Medical Services (JCEMS) will foster collaboration within the CBEMS community to build a culture of research and scholarship.

\section{Gap in the Literature}

The lack of research and evidence-based guidelines supporting clinical and policy decisions in pre-hospital emergency care has been repeatedly articulated. Relative to other healthcare fields and medical specialties, EMS is in its infancy; only in the 1960s and 70s did influential publications $s^{2,3}$ and impactful legislation ${ }^{4,5}$ carve a path for the development of modern EMS systems. Yet, as EMS systems grew, a vigorous research apparatus failed to develop. In 2001, the National EMS Research Agenda - published with the support of the National Highway Traffic Safety Administration and the Maternal and Child Health Bureau of the Health Resources Services Administration $\neg$ - identified a distinct lack of high quality, methodologically rigorous, EMS-related research within the domains of clinical care, systems design, and educational practice. ${ }^{6}$ Building upon the agenda, the Institute of Medicine's (IOM's) Committee on the Future of Emergency Care in the United States Health System published "Emergency Medicine at the Crossroads" in 2006. The report reiterated the lack of existing EMS-related research to guide improvements in patient outcomes, and also provided further recommendations to expand the evidence base supporting clinical interventions and systems development. ${ }^{7}$ Since the publication of the National EMS Research Agenda and the IOM's report, EMS-related research has expanded and fruitful efforts have been undertaken to translate research findings into practice through the development of evidence-based guidelines. ${ }^{8-13}$ In fact, the recent recognition of EMS as a distinct boarded medical subspecialty by the American Board of Medical Specialists in $2013^{14}$ highlights a respect for EMS as a distinct fund of scientific knowledge, primed for original research and other scholarly works. However, despite advancements in EMS research, there is a continued recognition that EMS trails far behind other medical fields with respect to research.

Of concern in the current editorial is the dearth of published original research and related scholarship (eg, case reports, clinical reviews, and perspectives pieces) in the field of CBEMS. Even relative to the lack of scholarship within the traditional EMS community, CBEMS-related scholarly work is scarce. To our knowledge, less than a dozen research papers, case studies, or other forms of scholarship that are specifically focused on CBEMS have been published in the scholarly literature (ie, academic journals). Most of these articles have been published in scholarly journals that specifically cater to EMS medical directors (Prehospital Emergency Care and Prehospital and Disaster Medicine) or career college health professionals (eg, Journal of American College Health), rather than CBEMS leaders, providers, or affiliated professionals. Even in popular sources (ie, magazines or websites), articles describing CBEMS only rarely surface. Certainly, research and scholarship focused on traditional pre-hospital emergency care is of relevance to the CBEMS community; nonetheless, the unique structure of CBEMS organizations and the particular environment within which CBEMS organizations operate underscore the need for focused research projects and a targeted source of literature.

CBEMS organizations differ in significant ways from traditional EMS services. CBEMS organizations are uniquely situated on university and college campuses, which are characterized by complicated physical layouts, ${ }^{1}$ frequent mass gathering events (eg, stadium sporting events, music festivals), ${ }^{15,16}$ and highly-populated, confined areas. ${ }^{1}$ Although a large-scale survey of CBEMS patient encounters has yet to be undertaken, it is widely understood that CBEMS organizations serve a challenging demographic. To wit, CBEMS organizations must simultaneously be prepared to care for college-aged individuals - who may present with distinct clinical, social, and psychological needs - as well as faculty, staff, and campus visitors of all ages. CBEMS organizations are licensed to provide care at the basic life support (BLS) or advanced life support (ALS) level; however, providers are typically undergraduate [or, less frequently, graduate] students who serve within an organization for no more than four years. ${ }^{1}$ Moreover, unlike traditional EMS services, CBEMS organizations generally receive oversight 
from campus health centers, campus safety departments, or student government bodies. ${ }^{1}$ Given the unique environmental, clinical, and organizational challenges inherent in the provision of on-campus emergency care, it may not always be appropriate or feasible to translate research and scholarship designed for traditional EMS services into the practices of CBEMS organizations.

\section{Opportunities for Growth}

The paucity of literature represents an exciting opportunity for the CBEMS community. CBEMS leaders and providers may now conduct original research and scholarship in an under-explored field, fostering the advancement of CBEMS while simultaneously promoting their own professional and scholarly development. By conducting research and preparing scholarly work for peer review, CBEMS leaders and providers can identify best practices to share with the entire community. Student-providers are in a particularly strong position to produce research and scholarship and to translate findings into practice. In contrast to traditional EMS agencies that operate outside of academic institutions, CBEMS organizations operate within colleges and universities designed to foster inquisition and investigation. Likely, the majority of CBEMS leaders and providers are already actively engaged in scholarly endeavors and aspire to pursue careers in intellectually rigorous fields. Thus, the CBEMS community represents a cohort of motivated, curious, and academically-minded individuals who are eager to both practice and advance medicine. In fact, the development of a cadre of CBEMS researchers may be a route to the promotion of research that extends beyond campuses into the broader field of EMS. Ultimately, while the lack of scholarship within the CBEMS community is cause for concern, this community is uniquely prepared to support scholarly endeavors.

\section{Challenges to Conducting Research}

While many barriers that hinder research efforts within traditional EMS communities across the world have been identified over the last two decades, ${ }^{6,717-23}$ the CBEMS community is well-positioned to overcome them. These barriers may be broadly grouped into seven domains: Data Collection, Ethical Conduct, Funding Sources, Competing Obligations, Continuity of Leadership, Education and Mentorship, and Culture and Values.

\section{Data Collection}

Collecting data on patient encounters is complicated by the fact that patients are often treated by providers from multiple EMS organizations and facilities, who may be reluctant to share data. In addition, for CBEMS organizations in particular, low call volumes create challenges for compiling enough data points to conduct studies with sufficient statistical power. Few efforts have been undertaken to systematically compile data from multiple CBEMS organizations, ${ }^{1,24,25}$ which may reflect a lack of standardization in data collection practices; organizations collect data via either pen-and-paper or electronic means and may not collect the same types of data points. In addition, organizations may inconsistently collect data points ${ }^{26}$ or may apply differing data definitions from each other (eg, defining an encounter with an intoxicated patient as either a medical or a behavioral call). Lastly, appropriate data analysis may require a consistent definition of CBEMS-specific patient encounters. For example, it remains to be determined whether CBEMS responses to off-campus incidents should be analyzed in aggregate with or separately from on-campus incidents.

Despite challenges in data collection, CBEMS providers are well positioned to methodically collect data by coupling their academic backgrounds with the opportunities provided by NCEMSF. Specifically, NCEMSF hosts an organizational database ${ }^{27}$ which has the potential to serve as a rich source of information on CBEMS organizations, provided the database is regularly updated by each organization. In addition, NCEMSF has developed a cardiac arrest response registry, ${ }^{28}$ which represents a preliminary model for large-scale, clinical-based data collection. Lastly, the regional coordinator system of NCEMSF provides a networking route for organizations to compile sufficient data to appropriately power studies.

\section{Ethical Conduct}

Prior to conducting studies on human participants to contribute to generalizable knowledge (ie, publishable research), approval from an Institutional Review Board (IRB) is required. Although IRBs may not be familiar with standard EMS practices, IRBs serve to ensure that research will be conducted ethically. For example, IRBs ensure that participants or patients will be capable of providing informed consent - or appropriately exempted - and that their privacy will be respected. Prior to 
submission of an application to an IRB, researchers must determine (1) when, how, and by whom informed consent must be obtained, (2) how to properly de-identify participant or patient information in compliance with HIPAA, and (3) how to properly obtain patient information from entities involved in the continued care of patients (eg, receiving hospitals). Obtaining informed consent in the pre-hospital, campus-based environment is complicated by the facts that patients may present with debilitating conditions and that students may be considered "vulnerable populations." Nonetheless, applying for IRB approval is commonplace at academic institutions, whereas a traditional EMS service may not even have readily available access to an IRB. Due to the stringent guidelines mentioned, preparing a proposal to an IRB should be seen as an opportunity to develop an appropriately- and ethically-designed study that can push collegiate EMS further in both research and patient care.

\section{Funding Sources}

Acquiring funding may be difficult because grants may be limited at the federal, state, and local levels, and because submitting applications for funding is time-consuming and, often, complicated. Thankfully, research funding is typically, and often easily, obtained from university-wide grants or from specific academic departments. Academic institutions often pride themselves on their support of student initiatives that supplement classroom education and promote student health and safety. Local and regional funding sources may also be interested in supporting CBEMS organizations within their jurisdiction, particularly organizations that respond to calls in the broader, off-campus community.

\section{Competing Obligations}

Research is often time-consuming, and may be seen as secondary in priority to clinical and administrative duties. CBEMS providers, however, are often ambitious individuals who are inclined to pursue multiple projects and responsibilities at any given time. These student-providers are not only motivated to be intensely involved in EMS, but also various extracurricular activities, jobs, and research within their respective academic disciplines. In fact, by conducting CBEMS-related research, student-providers may find a way to bridge their academic and EMS-related obligations and interests.

\section{Continuity of Leadership}

For organizations staffed primarily by students, annual or frequent staff turnover may hinder continuity of leadership throughout long-term research projects. Long-term research projects may also be interrupted by breaks in the academic calendar. Fortunately, CBEMS organizations are adept at transitioning clinical and administrative leadership duties over time, thereby affording the opportunity to link research and scholarly projects to existing systems of leadership transition. CBEMS organizations also typically receive oversight or supervision from professional staff and advisors who may provide continuity of support for research projects.

\section{Education and Mentorship}

A focus on research is frequently absent from standard EMS courses. As a result, providers often lack the requisite knowledge and technical expertise required to conduct methodologically rigorous EMS-related research studies on their own. In addition to a lack of familiarity with relevant research methodologies, providers may not know how to obtain Institutional Review Board approval, acquire funding, recruit collaborators and participants, or prepare their research for presentation or publication. CBEMS providers, however, are often deeply engaged with scholarly endeavors outside of EMS, offering a route for the development of relevant expertise. CBEMS providers also have access to faculty support within their respective academic institutions, as discussed later in the "Collaborative Research and Scholarship" section.

\section{Culture \& Values}

Perhaps the greatest barrier to producing CBEMS-related research and scholarship is the prevalence of a culture that does not prioritize, value, or recognize the need for scholarship. Most of the scarce CBEMS-related peer-reviewed articles [or published abstracts] have been produced by NCEMSF ${ }^{1,25}$ or Georgetown University, ${ }^{15,16,26,29-31}$ suggesting that conducting CBEMS-related research has not been a priority for most organizations and providers; research needs and opportunities are typically absent from most discussions about organizational development. As a result, CBEMS providers may not recognize the tightly-knit relationships between innovative research, organizational development, and improvements in patient care. However, despite the lack of a rich scholarly culture, 
the CBEMS community values personal growth, innovation, and service. Scholarship and research - when linked to improvements in practice - therefore strongly support the CBEMS community's values and commitments.

\section{Collaborative Research and Scholarship}

Many of these barriers can be overcome by exploiting one of the characteristics that best defines CBEMS: collaboration. Developing a culture of scholarship within the CBEMS community will require a dedicated effort to promote collaboration within CBEMS organizations; between CBEMS providers, professional clinicians, and academic faculty; and between multiple organizations.

Collaborative research fosters camaraderie and builds community. Collaborating with students from multiple disciplines is also essential to attacking research questions that do not fall neatly within one academic purview. The advancement of CBEMS is an inherently multidisciplinary pursuit that will require rigorous investigations of clinical practice, organizational management strategies, administrative and financial policies, educational methods, technological developments, ethical decision-making, provider mental health, and far more. Improving organizations through research and scholarship will require the involvement of students from many academic disciplines. For example, a study designed to identify the factors that lead to post-shift stress in a population of providers might benefit from the involvement of a student in the exercise science department (to help measure physiological stressors) and a student in the psychology department (to capture psychological stressors).

In addition to collaboration between students from multiple disciplines, collaboration with CBEMS' partners in care (ie, health centers, campus safety, and traditional EMS) and faculty are essential. Faculty members are able to draw upon their knowledge of various research methods and relevant processes (eg, applying for funding, submitting IRB proposals, and preparing publications). Students can pair their own enthusiasm toward a research problem with their faculty's thorough understanding of research to produce scholarly solutions that will improve their organization, campus, and community. Furthermore, because each organization should have a medical director, CBEMS scholars should have access to an in-house collaborator with an advanced medical license who is able to provide expertise on clin- ically-geared projects. As CBEMS-related research becomes widespread, organizations may even attract more research-minded students, faculty advisors, and medical directors, thereby allowing initial research efforts to pave a path for future work.

Inter-agency collaboration provides great opportunities to identify and approach large-scale initiatives and projects that affect a wider population of CBEMS providers, such as evaluating mass-casualty incidents during campus events. Multiple organizations working together may also take advantage of shared funding, resources, data, and knowledge, as well as a consolidation of manpower. Such inter-agency collaboration could especially benefit neighboring organizations that are similar in geography, demography, student population size, and state or regional protocols.

\section{JCEMS \& NCEMSF: Forging a Partnership} On its 25th Anniversary, NCEMSF formed a partnership with The Journal of Collegiate EMS (JCEMS) to promote CBEMS scholarship and to spark immediate and sustainable progress in this young, growing field of research. JCEMS will provide an open access, peer-reviewed journal edited by a board of established leaders, clinicians, and research scholars, with the goal of supporting evidence-based practice and strengthening the voice of the collegiate CBEMS community. The JCEMS Editorial Board will maintain editorial autonomy while NCEMSF - an established and enduring institution will provide the journal with networking, administrative, and financial support.

The rigorous standards demanded by the JCEMS peer-review process and ethical guidelines place JCEMS in line with the most respected academic journals. Peer review demands scholarly accountability through stringent evaluation, which facilitates impactful, professional collaboration and increases credibility. Peer review is accomplished by independent reviewers - diligently selected by the JCEMS Editorial Board - who serve as leaders and scholars in their fields, who practice at the top of their advanced degrees, and whose specialties align with the scope of each reviewed article. In addition to a rigorous peer review process, JCEMS freely provides all of its content online. As an open access journal, JCEMS provides a consolidated resource from which providers and affiliated professionals may seek current, evidence-based 
information to help them improve their organizations and, ultimately, patient outcomes.

In addition to providing a forum for CBEMS providers to publish, JCEMS seeks to make research more accessible to developing scholars. In collaboration with NCEMSF, JCEMS will help identify mentors who can help guide CBEMS providers and organizations in the development of research projects and the production of publishable work. By identifying mentors, JCEMS and NCEMSF will help individual organizations join in the effort to mount research as a pillar of the broader CBEMS community. Since its inception in 1993, NCEMSF has demonstrated to the EMS community that CBEMS organizations can provide emergency care of the highest quality. Now, it is time to establish a CBEMS scholarship-base worthy of the same respect.

\section{Acknowledgments}

We thank Ketan Yerneni for his insightful comments on the organization and content of the manuscript.

\section{References}

1. Fisher J, Ray A, Savett SC, Milliron ME, Koenig GJ. Collegiate-based emergency medical services (EMS): A survey of EMS systems on college campuses. Prehosp Disaster Med. 2006;21(2):91-96. https://doi.org/10.1017/ S1049023X00003411

2. National Academy of Sciences and National Research Council Committee on Trauma and Committee on Shock. Accidental Death and Disability: The Neglected Disease of Modern Society. Washington, DC: The National Academies Press; 1966. https://www.ncbi.nlm.nih.gov/books/NBK222962/. Accessed February 7, 2018.

3. Pantridge JF, Geddes JS. A mobile intensive-care unit in the management of myocardial infarction. Lancet. 1967;290(7510):271-273. https://doi.org/10.1016/S01406736(67)90110-9

4. Highway Safety Act. Public Law No. 89-564, 89 Stat 3052 (1966). https://www.gpo.gov/fdsys/pkg/STATUTE-80/pdf/ STATUTE-80-Pg731.pdf. Accessed February 7, 2018.

5. Emergency Medical Services Systems Act. Public Law No. 93154, 87 Stat 594 (1973). http:// www.gpo.gov/fdsys/granule/ STATUTE-87/STATUTE-87-Pg594/contentdetail.html. Accessed February 7, 2018.

6. U.S. National Highway Traffic Safety Administration, Department of Transportation and The Maternal and Child
Health Bureau, Health Resources Services Administration, Department of Health and Human Services. National EMS Research Agenda. https://www.nhtsa.gov/people/injury/ems/ ems-agenda/EMSResearchAgenda.pdf. Published December 31, 2001. Accessed February 7, 2018.

7. Committee on the Future of Emergency Care in the United States Health System, Institute of Medicine. Emergency Medical Services at a Crossroads. Washington, DC: The National Academies Press; 2006. https://doi. org/10.17226/11629

8. Lang ES, Spaite DW, Oliver ZJ, et al. A national model for developing, implementing, and evaluating evidencebased guidelines for prehospital care. Acad Emerg Med. 2012;19(2):201-209. https://doi.org/10.1111/j.15532712.2011.01281.x

9. Bulger EM, Snyder D, Schoelles K, et al. An evidence-based prehospital guideline for external hemorrhage control: American college of surgeons committee on trauma. Prehosp Emerg Care. 2014;18(2):163-173. https://doi.org/10.3109/10 903127.2014 .896962

10. Gausche-Hill M, Brown KM, Oliver ZJ, et al. An evidencebased guideline for prehospital analgesia in trauma. Prehosp Emerg Care. 2014;18(suppl 1):25-34. https://doi.org/doi:10.3 $109 / 10903127.2013 .844873$

11. Shah MI, MacIas CG, Dayan PS, et al. An evidence-based guideline for pediatric prehospital seizure management using grade methodology. Prehosp Emerg Care. 2014;18(suppl 1):1524. https://doi.org/10.3109/10903127.2013.844874

12. Martin-Gill C, Gaither JB, Bigham BL, Myers JB, Kupas DF, Spaite DW. National Prehospital Evidence-Based Guidelines Strategy: A Summary for EMS Stakeholders. Prehosp Emerg Care. 2016;20(2):175-183. https://doi.org/10.3109/1090312 7.2015.1102995

13. Thomas SH, Brown KM, Oliver ZJ, et al. An Evidence-based Guideline for the air medical transportation of prehospital trauma patients. Prehosp Emerg Care. 2014;18 (suppl 1):35-44. https://doi.org/10.3109/10903127.2013.844872

14. American Board of Emergency Medicine. EMS-Overview. https://www.abem.org/public/subspecialty-certification/ emergency-medical-services/ems-overview. Accessed February 7, 2018.

15. Stefos KA, Nable J V. Implementation of a high-performance cardiopulmonary resuscitation protocol at a collegiate emergency medical services program. J Am Coll Health. 2016;64(4):329-333. https://doi.org/10.1080/07448481.201 6.1138480 
16. Jeffery RM, Dickinson L, Ng ND, DeGeorge LM, Nable J V. Naloxone administration for suspected opioid overdose: An expanded scope of practice by a basic life support collegiatebased emergency medical services agency. J Am Coll Health. 2017;65(3):212-216. https://doi.org/10.1080/07448481.201 6.1277730

17. Tippett V. Clark M. Woods S. FitzGerald G. Towards a national research agenda for the ambulance and pre-hospital sector in Australia. J Emerg Prim Health Care. 2003;1(1-2). https://ajp.paramedics.org/index.php/ajp/article/view/73. Accessed February 7, 2018.

18. Leonard JC, Scharff DP, Koors V, et al. A qualitative assessment of factors that influence emergency medical services partnerships in prehospital research. Acad Emerg Med. 2012;19(2):161-173. https://doi.org/10.1111/j.15532712.2011.01283.x

19. Jensen JL, Bigham BL, Blanchard IE, et al. The Canadian National EMS research agenda: A mixed methods consensus study. Can J Emerg Med. 2013;15(2):73-82. https://doi. org/10.2310/8000.2013.130894

20. O’Meara P, Maguire B, Jennings P, Simpson P. Building an Australasian paramedicine research agenda: a narrative review. Health Res Policy Syst. 2015;13(1):79. https://doi.org/10.1186/ s12961-015-0065-0

21. Lerner EB, Weik T, Edgerton EA. Research in Prehospital Care: Overcoming the Barriers to Success. Prehosp Emerg Care. 2016;20(4):448-453. https://doi.org/10.3109/10903127.201 4.980480

22. van de Glind I, Berben S, Zeegers F, et al. A national research agenda for pre-hospital emergency medical services in the Netherlands: A Delphi-study. Scand J Trauma Resusc Emerg Med. 2016;24(1):2. https://doi.org/10.1186/s13049-0150195-y

23. Batt A, Knox S. Building research capacity among Irish prehospital practitioners. Irish J Paramed. 2017;2(2). http:// irishparamedicine.com/index.php/ijp/article/view/67. Accessed February 7, 2018.

24. King B, Zachariah B, Cone D, Clark P. A survey of emergency medical services systems on college and university campuses. Prehosp Disaster Med. 1996;11(4):265-268. https://doi. org/10.1017/S1049023X00043107

25. Wolbrom DH, Rahman A, Hilton MT. A Survey of 200 National Collegiate Emergency Medical Service Organizations. Prehosp Disaster Med. 2017;32(suppl 1):S169-S170. https:// doi.org/10.1017/S1049023X17004575
26. Katzer R, Barton DJ, Adelman S, Clark S, Seaman EL, Hudson KB. Impact of implementing an EMR on physical exam documentation by ambulance personnel. Appl Clin Inform. 2012;3(3):301-308. https://doi.org/10.4338/ACI2012-03-RA-0008

27. National Collegiate Emergency Medical Services Foundation. EMS Organization Database. https://www.ncemsf.org/ resources/ems-organization-database/groups-listed-by-region. Accessed February 7, 2018.

28. National Collegiate Emergency Medical Services Foundation. Collegiate EMS and Out-of-Hospital Cardiac Arrest. https:// www.ncemsf.org/resources/research/2-public-articles/ webpages/662-collegiate-ems-and-out-of-hospital-cardiacarrest. Accessed February 7, 2018.

29. Katzner R, Adelman S, Barton D, Clark S, Seaman E, Hudson K. The Use of a Quick Reference Card to Improve the Documentation of the Physical Exam by a Collegiate BLS EMS Organization. Georg Univ J Health Sci. 2012;6(1):20. https://blogs.commons.georgetown.edu/journal-of-healthsciences/issues-2/vol-6-no-1-december-2011/the-use-of-aquick-reference-card-to-improve-the-documentation-of-thephysical-exam-by-a-collegiate-bls-ems-organization/. Accessed February 7, 2018.

30. Davis JE, Churosh N, Borloz M, Howell J. Knowledge of SelfInjectable Epinephrine Technique Among Emergency Medical Services Providers. Ann Emerg Med. 54(suppl 3):S36. https:// doi.org/10.1016/j.annemergmed.2009.06.139

31. Barton DJ, Tift FW, Cournoyer LE, Vieth JT, Hudson KB. Acute Alcohol Use and Injury Patterns in Young Adult Prehospital Patients. Prehosp Emerg Care. 2016;20(2):206-211. https://doi.org/10.3109/10903127.2015.1076101

Author Affiliations: From The Journal of Collegiate Emergency Medical Services, Philadelphia, PA, USA (N.M.G.F, B.J.D., J.V.N.); Physician Assistant Program, Drexel University College of Nursing and Health Professions, Philadelphia, PA, USA (B.J.D.); Georgetown Emergency Response Medical Service, Georgetown University, Washington, DC, USA (J.V.N.); Department of Emergency Medicine, MedStar Georgetown University Hospital, Georgetown University School of Medicine, Washington, DC, USA (J.V.N.); National Collegiate Emergency Medical Services Foundation, West Stand Lake, NY, USA (G.J.K.); Department of Surgery, Division of Acute Care Surgery, Thomas Jefferson University School of Medicine, Philadelphia, PA, USA (G.J.K.).

Address for Correspondence: Nicholas M.G. Friedman, BA, EMT -B, The Journal of Collegiate Emergency Medical Services, 117 N 15 ${ }^{\text {th }}$ St., Philadelphia, PA 19102, USA. E-mail: JCEMS@CollegeEMS.com 
Conflicts of Interest/Funding Sources: By the JCEMS Submission Declaration Form, all authors are required to disclose all potential conflicts of interest and funding sources. N.M.G.F, B.J.D., and J.V.N. serve in uncompensated editorial and/or staff roles for JCEMS. G.J.K. serves as the uncompensated President of NCEMSF. All authors declared that they have no others conflicts of interest. All authors declared that they did not receive funding to conduct the research and/or writing associated with this work.

Authorship Criteria: By the JCEMS Submission Declaration Form, all authors are required to attest to meeting the four ICMJE.org authorship criteria: (1) Substantial contributions to the conception or design of the work; or the acquisition, analysis, or interpretation of data for the work; AND (2) Drafting the work or revising it critically for important intellectual content; AND (3) Final approval of the version to be published; AND (4) Agreement to be accountable for all aspects of the work in ensuring that questions related to the accuracy or integrity of any part of the work are appropriately investigated and resolved.

Submission History: Received February 7, 2018; accepted for publication February 7, 2018.

Published Online: February 23, 2018

Published in Print: February 23, 2018 (Volume 1: Issue 1)

Reviewer Information: In accordance with JCEMS editorial policy, Editorial manuscripts are reviewed by the JCEMS Editorial Board. Editorial manuscripts are published as submitted - save for copy-editing - by the Editor-in-Chief or designee. JCEMS thanks the Editorial Board members who contributed to the review of this work.

Copyright: (C) 2018 Friedman, Dingler, Nable, \& Koenig. This is an open access article distributed under the terms of the Creative Commons Attribution 4.0 International (CC BY 4.0) License, which permits unrestricted use, distribution, and reproduction in any medium, provided the original author and source are credited. The full license is available at: https://creativecommons.org/licenses/by/4.0/

Electronic Link: https://doi.org/10.30542/JCEMS.2018.01.01.01 